\title{
The Association between Foot Morphology and Dynamic Balance Performance as Measured by the Star Excursion Balance Test
}

\author{
Lubetzky V. Anat ${ }^{1 *}$ and Kramer A. Patricia ${ }^{2}$ \\ ${ }^{1}$ Department of Physical Therapy, New York University, New York, New York \\ ${ }^{2}$ Departments of Anthropology and Orthopaedics, University of Washington, Seattle, Washington
}

Received: April 03, 2015; Accepted: July 22, 2015; Published: September 09, 2015

*Corresponding author: Anat Lubetzky, 380 2nd Avenue, 4th floor, New York, NY 10010, USA, Tel: 001- 212- 998-9195; Email: anat@nyu.edu

\begin{abstract}
The purposes of this descriptive study were to explore the association between foot morphology, severity of past injuries and Star Excursion Balance Test (SEBT) performance in recreational runners. We interviewed 20 healthy recreational runners (aged 23-58 years) about their running habits and history of injuries. We calculated arch height index, a measure of foot morphology, from their static footprint. Participants performed the SEBT. Participants with lower medial longitudinal foot arch reached significantly farther on all SEBT directions except for anterolateral. The Spearman correlation (Rs) between arch index and those 7 directions ranged from 0.455 to 0.617 ( $p$ values ranging from 0.01 to 0.05 ). Severity of injuries in the past was not associated with SEBT performance. Assessing parameters that are associated with balance performance can help therapists to understand the underlying impairments leading to decreased or enhanced dynamic balance performance. Researchers and clinicians need to be aware of potential differences in dynamic balance performance in individuals with different foot morphologies.
\end{abstract}

Keywords: Star Excursion Balance Test; Dynamic Balance; Arch Index; Running; Injuries

\section{Introduction}

Balance is complex, and there are several underlying factors that can explain improved or decreased balance performance. Interventions aimed to enhance postural control ability and prevent falls should be directed at the specific diagnosis of the underlying impairment(s) causing the postural control dysfunction. A specific intervention is expected to improve balance performance more than a 'general balance training' approach [1]. One such potential parameter that may contribute to balance performance is foot morphology. The foot provides a narrow base of support on which the body maintains balance. From a biomechanical perspective, it is plausible to expect that variation in the morphology of the foot may influence postural control in general and dynamic balance in particular [2].

The Star Excursion Balance Test (SEBT) is a reliable clinical test [3], originally designed to assess lower-extremity balance and neuromuscular control [4]. The protocol is easily implemented in the field and clinic, requiring no specialized equipment or advanced training. In addition, the SEBT is challenging enough to stress the postural control system, making it an ideal task to be used by athletic trainers and physical therapists to assess balance within athletic populations $[3,5]$.

The association between dynamic balance, as measured by the SEBT, and foot morphology was investigated in 2 studies with mixed results [2,6]. On one hand, Gribble and Hertel [6] found no significant relationships between foot types and reach distances on the SEBT using rearfoot and forefoot angles (classified as pes planus, pes rectus, and pes cavus) in 30 recreationally active participants. They concluded that foot type was not an important covariate of balance performance. On the other hand, Cote et al. [2] found differences in reach distance on a few SEBT directions as a function of foot type in 16 healthy individuals when foot type was determined using the navicular drop method (classified as pronated, neutral or supinated). They suggest that postural stability is affected by foot morphology and that foot type needs to be controlled for when measuring balance performance [2]. These differing results suggest that the association between dynamic balance performance and foot morphology is not well understood.

In addition to morphology, another factor that may be important in order to interpret dynamic balance performance is history of injuries. While ankle sprains [4] and ACL deficiencies [7] were found to impact performance on the SEBT among recreationally active participants, the effect of previous injury, in general, has not been investigated. Given that almost every epidemiological study of running has identified previous injury (usually defined as an injury occurring in the 12 months prior to the study) as a risk factor for future injuries $[8,9]$, previous injury seems to be an important characteristic of recreationally active persons, especially runners. The mechanism that links past and future injuries, however, still needs clarification [10]. Impaired dynamic balance performance is one potential underlying 
explanation for the strong association between previous injury and recurrence of injuries. In light of these findings, our purposes were to explore the association between foot morphology, severity of injuries in the past, and normalized SEBT reach distance in recreational runners.

\section{Methods}

Participants in this study were recreational runners who ran regularly at least once a week (indoors or outdoors) and were currently injury free and able to run. Adult participants aged 18-60 years, of any ethnicity and sex were eligible for inclusion. Professional runners (defined as runners who receive payment and /or sponsorship in order to train or compete) were excluded from the study. Our sample of convenience was recruited through flyers posted around the University of Washington. The methods used for this study conform to the Code of Ethics of the declaration of Helsinki and the United States Federal Regulations. This study was approved by our institution's Institutional Review Board and a written informed consent was obtained from all participants.

Following the informed consent process, participants answered questions from a structured interview, and their anthropometric measurements, as well as static footprint, were taken. Participants then performed the Star Excursion Balance Test (SEBT). Rest breaks were periodically offered yet none of the participants needed a break during the session.

The structured interview was conducted to identify running habits and sports-related injuries in the past. We asked the participants, on average, how many times per week they typically ran, the average length of each of their running sessions in time and distance (minutes and miles), and on what type of surface they typically ran. A sports injury was defined according to the recommendation made by the Council of Europe as "any injury occurring as a result of sports activity and causing one or more of the following: the subject had to stop sports activity and/or could not fully participate in the next planned sports activity and/or could not go to work the next day and/or needed medical attention and /or suffered from pain or stiffness during 10 subsequent days while participating in sports" [11]. Injury report included type of injury and its anatomic location according to a modification of the "Barell body region by nature of injury diagnosis matrix" [12]. The Barell matrix, designed by the Israeli Ministry of Health and a special medical team of the Israeli Defense Forces, provides information regarding anatomic locations of injuries (e.g. head and neck, spine and back etc.) as well as their diagnosis (e.g. fracture, dislocation, internal, open wound, amputations etc.). We used a modification of the Barell matrix [13] including only the musculoskeletal type of injuries (i.e. contusion, fracture, dislocation, ligament sprain, muscle strain, nonspecific). In addition, because our purpose was to explore the association between history of injury and dynamic balance in recreational runners, we focused on lower extremity injuries. For the modified Barell matrix, see Appendix A. For each reported injury, participants were asked whether the injury occurred in the last 12 months or before that, and whether they needed to change their activity habits permanently as a result of the injury.

Information from the interview was put into 6 weighted categories that were summarized into a total severity score. Those categories include: type of injury [14], timing of injury, recurrence, injury rate (defined as number of injuries divided by age and multiplied by 100), activity change as a result of injury, and surgical procedures required. Content validity [15] for the choice of categories as well as the weight of each category was obtained from 8 physical therapists and physicians all with at least 5 years of experience in sports-related injuries rehabilitation and who are licensed to practice either in Israel or in the United States. All of our experts agreed that that the categories reflected severity (i.e. that they would expect that people with a higher score would have greater tissue damage and/or decreased function as a result of their sport-related injuries). In addition, the final weights of the different categories were adjusted based on the experts' suggestions. A full description of our severity assessment appears in Appendix B.

We measured mass, standing height, and seated height. We calculated limb length by subtracting the participant's seated height from his/her standing height. We used a pressure sensitive mat and associated software (Foot scan USB plate, RSScan International, Olen, Belgium) to obtain the static footprints of all participants. The $0.5 \times 0.4 \mathrm{~m}$ pressure-sensitive mat sends readings taken at $300 \mathrm{~Hz}$ from 4096 sensor areas to software which produces the footprint images. We asked the participants to stand comfortably with bare feet on the pressure mat with their typical weight distribution while a snap-shot of their footprint was taken. To quantify foot morphology, we used the arch height index measure because it is a simple and reliable assessment of arch height [16]. Arch index is defined as: $\mathrm{B} / \mathrm{A}+\mathrm{B}+\mathrm{C}$, when $\mathrm{A}=$ rearfoot area, $\mathrm{B}=$ midfoot area, and $\mathrm{C}=$ forefoot area, excluding the toes [6]. Higher arches have lower arch indices. Figure 1 demonstrates how we calculated arch height index from the static footprint obtained with the RSScan program.

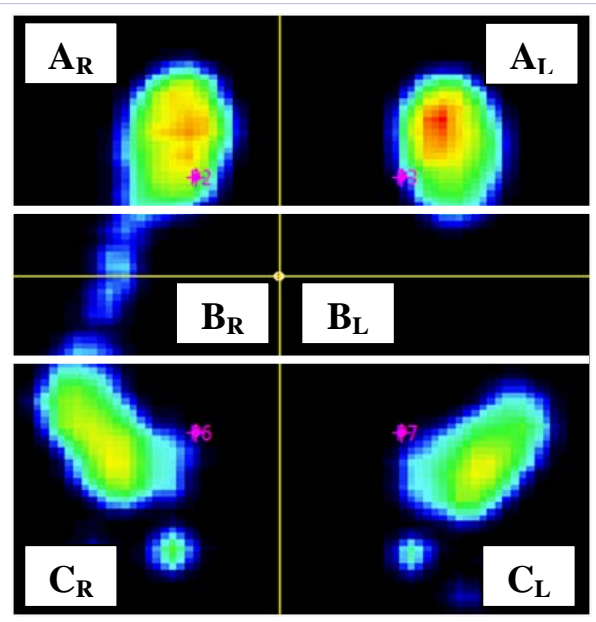

Figure 1: Calculation of Arch Index from a participant's static footprint. Arch height index is defined as: $B_{R} / A_{R}+B_{R}+C_{R}$ for the right foot and $B_{L}$ $/ A_{L}+B_{L}+C_{L}$ for the left foot. 
Each participant performed the SEBT protocol as described by Hertel et al. [3]. The participant stood at the center of a grid placed on the floor, with 8 lines extending at $45^{\circ}$ increments from the center of the grid. Participants stood on one leg with their hands on the hips while reaching with the contralateral leg as far as possible along a specific direction, defined as: anterolateral $(\mathrm{AL})$, anterior $(\mathrm{A})$, anteromedial $(\mathrm{AM})$, medial $(\mathrm{M})$, posteromedial $(\mathrm{PM})$, posterior $(\mathrm{P})$, posterolateral (PL), and lateral (L) relative to the stance leg. Testing of both legs started with 6 practice trials in each of the 8 directions to become familiar with the task and minimize learning effects on the measurement, as recommended by Hertel et al. [3]. As the participant lightly touched the farthest point possible on the line, the examiner marked this point. The participant then returned to a bilateral stance and rested for 15 seconds before performing the next reach. The order of the directions was randomized for each participant and 3 reaches in each direction were recorded. The examiner then manually measured the distance from the center of the grid to the touch point with a tape measure graded in centimeters.

Descriptive statistics of the sample for the variables age, weight, height, limb length, and running pattern (duration, and distance per session and weekly frequency) were calculated. We calculated the mean of the 3 trials for each SEBT direction and normalized it by dividing by limb length as suggested by Gribble and Hertel [6]. To compare each of the eight excursions (averaged and normalized) distances of the right and left limbs of participants we used related samples non parametric comparisons (Wilcoxon signed rank tests). We also calculated the mean normalized reach in all 8 directions to provide an SEBT composite score [17]. Because the distribution of the mean arch index is skewed to the left ( $\mathrm{arch}$ index is truncated at $0=$ no midfoot contact), we used Wilcoxon signed rank tests to compare the arch index of both feet. Spearman correlations were calculated to determine the relationship between each SEBT direction reach distance and arch index, each SEBT direction reach distance and severity of injuries in the past and. Because of the exploratory nature of this study, alpha level was set at 0.05 . All calculations were done using SPSS version 18.0.

\section{Results}

Our sample included 15 women and 5 men with mean age of 33.45 years (SD 10.93, minimum 23, maximum 58). The average body mass was $68.82 \mathrm{~kg}$ (SD 12.82) and average height 169.68 cm (SD 6.88). Participants ran 3.6 times a week on average (range 2-8) with mean duration of running session 47.13 minutes (range 26.5-80) and mean distance per session 5.03 miles (range 1.5-13.5). All of the participants were outdoor runners. Four participants also ran indoors occasionally.

No significant differences were identified between the limbs' reach distance ( $p$ values ranged from 0.135 to 0.936 ), or arch index $(P=0.91)$, hence data from the right and left limb were averaged.

The arch height index was found to be moderately and significantly correlated with 7 out of the 8 star reach directions.
Specifically, participants with higher arch index values (i.e., lower medial longitudinal foot arch) reached farther on all directions except for the AnteroLateral (AL) direction. The Spearman correlation coefficients (Rs) with their corresponding $\mathrm{R}^{2}$ and $p$ values appear in Table 1 and the relationship between arch index and the SEBT composite score is presented in Figure 2.

No statistically significant correlations were found between any SEBT direction and severity of past injuries. The Spearman correlation coefficients between SEBT and severity revolved around 0 , with the highest being $0.262(P=0.256)$.

\section{Discussion}

The role of foot morphology in static and dynamic balance performance has received little attention in the literature and previous investigations that included foot morphology were inconclusive. We, however, found a consistent relationship between foot morphology, as measured by the arch height index, and normalized SEBT reach distance. People with lower arches reached farther on all but one SEBT direction and had a statistically significantly higher SEBT composite score. Understanding why people with flat feet (lower arches) reached farther should be a target of future investigation. We hypothesize that ligamentous laxity in the feet and ankles of the participants with flat feet as compared to high arched feet [18] may lead to increased range of motion and increased reach distance. Another potential explanation is that flat feet provide larger base of

\begin{tabular}{|c|c|c|c|}
\hline \multicolumn{4}{|c|}{ Table 1: Correlation coefficients: SEBT and Arch Index. } \\
\hline Direction & R Spearman & $\boldsymbol{p}$ & $\mathbf{R}^{2}$ \\
\hline Anterior & 0.537 & 0.02 & 0.15 \\
\hline Anteromedial & 0.617 & 0.01 & 0.26 \\
\hline Medial & 0.492 & 0.03 & 0.26 \\
\hline Posteromedial & 0.564 & 0.01 & 0.35 \\
\hline Posterior & 0.489 & 0.03 & 0.24 \\
\hline Posterolateral & 0.488 & 0.03 & 0.27 \\
\hline Lateral & 0.455 & 0.05 & 0.25 \\
\hline Anterolateral & 0.281 & 0.24 & 0.06 \\
\hline
\end{tabular}

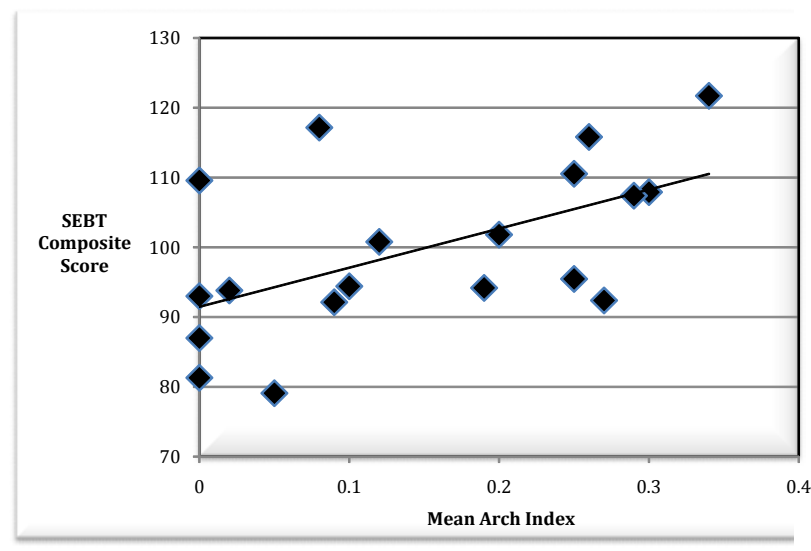

Figure 2: SEBT Composite Score and Mean Arch Index. 
support (i.e., greater area of the foot contacts the ground) and can accommodate to the surface better than do high arched feet.

Measuring static one leg stance with eyes open, Hertel at al. [3] found that healthy individuals with high arched feet had a significantly larger sway area than individuals with normal feet [19]. Tsai et al. [20] tested the center of pressure displacement of young adults standing on one leg with eyes closed. They found that, compared to participants with normal arch shape, individuals with high arches swayed more in both the antero-posterior and medio-lateral directions whereas individuals with low arches swayed more only in the antero-posterior direction. Cote et al. [2] however found no difference in postural sway as a function of foot type when participants were standing on one leg with eyes open or closed but sway variability was greater in participants with pronated (flat) feet than those with supinated (high arched) feet. Likewise, Karthikeyan et al. [21] found no relationship between center of pressure measures in single leg stance with eyes open or closed and forefoot angle. For SEBT performance, Hertel and Gribble [6], found no association between foot type and reach distance while Cote et al. found some effect of foot type on reach distance. Specifically, participants with pronated feet reached farther on the anterior and antero-medial directions and participants with supinated feet reached farther in the posterior and postero-lateral directions [2]. We found that arch height index was associated with reach distance in all but the anterolateral direction.

One possible explanation for the different finding regarding the relationship between foot morphology and reach distance is that each study used different methods to assess foot morphology. The degree to which a foot is arched can be assessed in many different ways that are each useful in particular clinical and/or research circumstances. Hertel and Gribble [6] used a method that uses static rearfoot and forefoot angles (i.e., the orientations of the heel and metatarsal heads relative to the ground) to classify foot type, while Cote et al. [2] used a technique where the change in arch height (i.e., distance from ground to navicular) was assessed between neutral and loaded (i.e., fully weight bearing) conditions. These 2 measures are not necessarily correlated [18] because they measure different aspects of foot morphology (e.g. rearfoot and forefoot vs. midfoot and unloaded neutral vs. fully loaded). We used the arch height index, which is the ratio of midfoot to total foot area derived from static weight-bearing footprints, because we were particularly interested in the relationship between the ground and the sole of the stationary foot. In addition, we kept the arch height index as a continuous measure rather than classifying it to three foot types.

Our findings of reduced reach distance in people with high arches (lower arch index) could be explained by the association between the supinated foot and hypomobility of the foot joints [22]. In addition, the supinated foot has less plantar contact area compared to flat or neutral foot which decreases the base of support from which the movement is performed and could lead to decreased sensory input regarding joint position [23,24]. In contrast, the hypermobile pronated foot could increase the ability to reach, especially in the medial direction and lead to overall improved performance when dynamic balance is measured by reach distance. This could explain why the only reach direction where we did not identify a relationship between reach distance and arch index was the anterolateral one.

Our study of recreational runners was designed to be descriptive with broad inclusion and exclusion criteria, which led to a diverse sample in terms of sex and age, including young as well as middle age adults. Previous studies indicate, however, that performance on the SEBT is expected to be fairly consistent between men and women when the reach distance has been normalized to leg length [25]. Also, while changes in postural control in the elderly are well documented [26], they are less established for young adults and middle-aged adults [27]. Yet Bouillon and Baker [28] found differences in reach distance on the SEBT between young and middle-age recreationally active women but our study was not powered to test for these differences. While our sample size was small, the moderate, significant relationship we found was consistent for 7 out of 8 reach directions which decreases the likelihood of spurious statistical findings.

Because all of the participants sustained injuries in the past, our findings can only be generalized to recreational runners who have sustained sports injuries in the past. The summary score we used to quantify severity of past injuries has not been used before. Despite its novelty, all of our experts agreed that the severity score used in our study provides more information than simply counting number of injuries in the past. Note that the severity score was highly correlated with number of injuries in the past (data not presented). In addition, injuries were selfreported and were, thus, subject to recall bias. We focused on an instrumented foot morphology measure and did not explore other kinematic, kinetic or personal factors that may explain dynamic balance performance. Hoch et al. [29] for example, found an association between forward reach on the SEBT and weight bearing ankle dorsiflexion range. In addition, we did not perform a clinical assessment of the foot posture, but instead used a simple and reliable instrumented assessment ( $\operatorname{arch}$ index). Future, larger studies using force plate data and motion analysis to analyze SEBT performance may shed light on the underlying biomechanical explanation for the association between foot morphology and SEBT performance.

\section{Conclusion}

In conclusion, we found positive, moderate and significant association between SEBT reach distance and foot morphology on 7 out of 8 SEBT directions among recreational runners. The lower the medial longitudinal arch was, the farther participants reached. Self-reported history of injuries was not correlated with SEBT performance.

- Practical Implications: Interventions aimed to enhance balance should be directed at the specific underlying impairment(s) causing the postural control dysfunction. Clinicians and researchers need to be aware that differences in foot morphology may affect dynamic balance ability. 
- In the clinic, if people have asymmetric foot morphology (i.e., one foot with a low arch and one foot with a high arch) the differences between their feet may affect their symmetry in performance of the SEBT.

- In the research setting, when examining dynamic balance performance via the SEBT, foot morphology may need to be adjusted for when comparing groups.

\section{References}

1. Horak FB. Postural orientation and equilibrium: what do we need to know about neural control of balance to prevent falls? Age Ageing. 2006; 35 Suppl 2:ii7-ii11. doi:10.1093/ageing/afl077.

2. Cote KP, Brunet ME, Gansneder BM, Shultz SJ. Effects of Pronated and Supinated Foot Postures on Static and Dynamic Postural Stability. J Athl Train. 2005; 40(1):41-6.

3. Hertel J, Miller SJ, Denegar CR. Intratester and intertester reliability during the Star Excursion Balance Tests. J Sport Rehabil. 2000; 9(2):104-16.

4. Olmsted LC, Carcia CR, Hertel J, Shultz SJ. Efficacy of the Star Excursion Balance Tests in Detecting Reach Deficits in Subjects With Chronic Ankle Instability. J Athl Train. 2002; 37(4):501-6.

5. Sabin MJ, Ebersole KT, Martindale AR, Price JW, Broglio SP. Balance performance in male and female collegiate basketball athletes: influence of testing surface. J Strength Cond Res. 2010; 24(8):2073-8. doi:10.1519/JSC.0b013e3181ddae13.

6. Gribble PA, Hertel J. Considerations for Normalizing Measures of the Star Excursion Balance Test. Meas Phys Educ Exerc Sci. 2003; 7(2):89100.

7. Herrington L, Hatcher J, Hatcher A, McNicholas M. A comparison of Star Excursion Balance Test reach distances between ACL deficient patients and asymptomatic controls. Knee. 2009; 16(2):149-52. doi:10.1016/j.knee.2008.10.004.

8. Macera CA. Lower extremity injuries in runners. Advances in prediction. Sports Med. 1992; 13(1):50-7.

9. Taunton JE, Ryan MB, Clement DB, McKenzie DC, Lloyd-Smith DR, Zumbo BD. A prospective study of running injuries: the Vancouver Sun Run “In Training” clinics. Br J Sports Med. 2003; 37(3):239-44.

10.van Mechelen W. Running injuries. A review of the epidemiological literature. Sports Med. 1992; 14(5):320-35.

11.Van Mechelen W, Twisk J, Molendijk A, Blom B, Snel J, Kemper HC. Subject-related risk factors for sports injuries: a 1-yr prospective study in young adults. Med Sci Sports Exerc. 1996; 28(9):1171-9.

12. Barell V, Aharonson-Daniel L, Fingerhut LA, Mackenzie EJ, Ziv A, Boyko V, Abargel A, et al. An introduction to the Barell body region by nature of injury diagnosis matrix. Inj Prev. 2002; 8(2):91-6.

13. Lubetzky-Vilnai A, Carmeli E, Katz-Leurer M. Prevalence of injuries among young adults in sport centers: relation to the type and pattern of activity. Scand J Med Sci Sports. 2009; 19(6):828-33. doi:10.1111/ j.1600-0838.2008.00854.x.
14. Goulet C, Hagel BE, Hamel D, Légaré G. Self-reported skill level and injury severity in skiers and snowboarders. J Sci Med Sport. 2010; 13(1):39-41. doi:10.1016/j.jsams.2008.10.002.

15. Streiner DL, Norman GR. Health Measurement Scales: A Practical Guide to Their Development and Use. 4th ed. Oxford: Oxford University Press; 2008.

16. Cavanagh PR, Rodgers MM. The arch index: a useful measure from footprints. J Biomech. 1987; 20(5):547-51.

17. Filipa A, Byrnes R, Paterno MV, Myer GD, Hewett TE. Neuromuscular training improves performance on the star excursion balance test in young female athletes. J Orthop Sports Phys Ther. 2010; 40(9):551-8. doi:10.2519/jospt.2010.3325.

18. Kanatli U, Gözil R, Besli K, Yetkin H, Bölükbasi S. The relationship between the hindfoot angle and the medial longitudinal arch of the foot. Foot Ankle Int. 2006; 27(8):623-7.

19. Hertel J, Gay MR, Denegar CR. Differences in Postural Control During Single-Leg Stance Among Healthy Individuals With Different Foot Types. J Athl Train. 2002; 37(2):129-32.

20. Tsai LC, Yu B, Mercer VS, Gross MT. Comparison of different structural foot types for measures of standing postural control. J Orthop Sports Phys Ther. 2006; 36(12):942-53. doi:10.2519/jospt.2006.2336.

21. Karthikeyan G, Jadav Jayraj S, Narayanan V. Effect of forefoot type on postural stability - a cross sectional comparative study. Int J Sports Phys Ther. 2015; 10(2):213-24.

22. Donateli R. The Biomechanics of the Foot and Ankle. Philadelphia: F.A. Davis; 1996.

23. Franco AH. Pes cavus and pes planus. Analyses and treatment. Phys Ther. 1987; 67(5):688-94.

24. Maki BE, Perry SD, Norrie RG, McIlroy WE. Effect of facilitation of sensation from plantar foot-surface boundaries on postural stabilization in young and older adults. J Gerontol A Biol Sci Med Sci. 1999; 54(6):M281-7.

25. Gribble PA, Hertel J, Plisky P. Using the Star Excursion Balance Test to assess dynamic postural-control deficits and outcomes in lower extremity injury: a literature and systematic review. J Athl Train. 2012; 47(3):339-57. doi:10.4085/1062-6050-47.3.08.

26. Sumway-Cook A, Woollacott M. Motor Control: Theory and Practical Applications. Philadelphia: Williams and Wilkins; 2001.

27.Wiesmeier IK, Dalin D, Maurer C. Elderly Use Proprioception Rather than Visual and Vestibular Cues for Postural Motor Control. Front Aging Neurosci. 2015; 7:97. doi:10.3389/fnagi.2015.00097.

28. Bouillon LE, Baker JL. Dynamic Balance Differences as Measured by the Star Excursion Balance Test Between Adult-aged and Middle-aged Women. Sports Health. 2011; 3(5):466-9. doi:10.1177/1941738111414127.

29. Hoch MC, Staton GS, McKeon PO. Dorsiflexion range of motion significantly influences dynamic balance. J Sci Med Sport. 2011; 14(1):90-2. doi:10.1016/j.jsams.2010.08.001. 


\begin{tabular}{|c|c|c|c|c|c|c|c|c|}
\hline $\begin{array}{l}\text { Appendix A. } \\
\text { In the table b } \\
\text { causing one o } \\
\text { a) Yo } \\
\text { b) Yo } \\
\text { c) Yo } \\
\text { d) Yo } \\
\text { e) Yo }\end{array}$ & $\begin{array}{l}\text { Modified Bare } \\
w \text { mark body sit } \\
\text { ore of the follow } \\
\text { d to stop sports } \\
\text { uld not fully par } \\
\text { uld not go to wo } \\
\text { eeded medical at } \\
\text { ffered from pair }\end{array}$ & $\begin{array}{l}\text { atrix (Barel } \\
\text { ou have prev } \\
\text { vity and/or } \\
\text { ate in the ne } \\
\text { he next day a } \\
\text { ion and/or } \\
\text { tiffness duri }\end{array}$ & $\begin{array}{l}\text { al. [12]) } \\
\text { isly injured } \\
\text { planned sp } \\
\text { /or } \\
10 \text { subsequ }\end{array}$ & $\begin{array}{l}\text { nd the diagnos } \\
\text { s activity and/ } \\
\text { tt days while } \mathrm{p}\end{array}$ & (if applica & For th & ose of this st & njury is defined as \\
\hline \multirow{4}{*}{ Spine } & Body site & Contusion & Fracture & Dislocation & $\begin{array}{l}\text { Ligament } \\
\text { sprain }\end{array}$ & $\begin{array}{l}\text { Muscle } \\
\text { strain }\end{array}$ & Non-specific & $\begin{array}{l}\text { In the last } 12 \\
\text { months/ before }\end{array}$ \\
\hline & Cervical spine & & & & & & & \\
\hline & Thoracic spine & & & & & & & \\
\hline & Lumbar spine & & & & & & & \\
\hline \multirow{4}{*}{ Trunk } & Rib cage & & & & & & & \\
\hline & Abdomen & & & & & & & \\
\hline & Pelvis & & & & & & & \\
\hline & Buttocks & & & & & & & \\
\hline \multirow{7}{*}{$\begin{array}{c}\text { Lower } \\
\text { Extremity }\end{array}$} & Hip joint & & & & & & & \\
\hline & Thigh & & & & & & & \\
\hline & Knee & & & & & & & \\
\hline & Shin & & & & & & & \\
\hline & Ankle & & & & & & & \\
\hline & Foot & & & & & & & \\
\hline & Toes & & & & & & & \\
\hline other & & & & & & & & \\
\hline
\end{tabular}

\section{Appendix B. Severity Assessment of Past Injuries}

\section{Category A: Type of injury}

Fractures/dislocation are more severe than other soft tissue injuries (based on Goulet et al. [16]):

3 points per intra-joint fracture / dislocation

2 points for fracture that does not involve the joint/stress fracture/ disc herniation/ subluxation

0 points for any other injury

\section{Category B: Timing}

Injury in the last 12 months weighs more than an injury occurring before the last 12 months:

1 point for any injury occurring in the last 12 months

0 points if none

\section{Category C: Recurrence}

1 point for each recurrence (the same injury happening repeatedly) up to 4 points

0 points if none

\section{Category D: Injury rate}

(Number of injuries/years of age)*100

\section{Category E: Activity change}

Injury that caused a permanent change in activity weighs more than injury which did not lead to a change in activity

3 points per injury that caused to stop the activity permanently

2 points for decreasing activity load

1 point for any other change

0 if none

\section{Category F: Surgery}

Injuries that required surgical procedure weigh more than injuries which did not

2 points per injury that required a surgery 
0 if none

Example of calculation for one participant:

Type of injury: This participant sustained 1 rib cage dislocation ( 3 p), 1 intra-joint fracture ( $3 p)$ and 2 fractures that did not involve the joint ( $2 \mathrm{X} 2$ p), the remaining injuries were nonspecific. Score $=10$ points

Injury in the last 12 months: Only 1 injury was sustained in the last 12 months. Score $=1$ point.

Recurrence: none of the injuries reoccurred. Score $=0$ points.

Injury rate: (6 injuries / 24 years of age) $* 100=25$

Activity change: The participant completely stopped one of her sporting activities as a result of an injury. Score $=3$

Surgical procedure: None of the injuries required a surgical intervention. Score $=0$

Total score $=10+1+0+25+3+0=39$ 